\title{
In vitro neuroprotective activities of two distinct probiotic consortia
}

\author{
D.R. Michael ${ }^{1 "}$, T.S. Davies ${ }^{1}$, K.E. Loxley ${ }^{1}$, M.D. Allen ${ }^{1}$, M.A. Good ${ }^{2}$, T.R. Hughes ${ }^{3}$ and S.F. Plummer ${ }^{1}$ \\ ${ }^{1}$ Cultech Limited, Unit 2 Christchurch Road, Baglan Industrial Park, Port Talbot, SA12 7BZ, United Kingdom; ${ }^{2}$ School of \\ Psychology, Tower Building, Cardiff University, Cardiff CF10 3AT, United Kingdom; ${ }^{3}$ Systems Immunity Research Institute, \\ Henry Welcome Building, Cardiff University, Cardiff CF144XXN, United Kingdom; darynm@cultech.co.uk
}

Received: 24 August 2018 / Accepted: 11 December 2018

(C) 2019 Wageningen Academic Publishers

OPEN ACCESS CC) RESEARCH ARTICLE

\begin{abstract}
Neurodegeneration has been linked to changes in the gut microbiota and this study compares the neuroprotective capability of two bacterial consortia, known as Lab4 and Lab4b, using the established SH-SY5Y neuronal cell model. Firstly, varying total antioxidant capacities (TAC) were identified in the intact cells from each consortia and their secreted metabolites, referred to as conditioned media (CM). 3-(4,5-dimethylthiazol-2-yl)-2,5-diphenyltetrazolium bromide (MTT) and Crystal Violet (CV) assays of cell viability revealed that Lab4 CM and Lab4b CM could induce similar levels of proliferation in SH-SY5Y cells and, despite divergent TAC, possessed a comparable ability to protect undifferentiated and retinoic acid-differentiated cells from the cytotoxic actions of rotenone and undifferentiated cells from the cytotoxic actions of 1-methyl-4-phenylpyridinium iodide (MPP+). Lab4 CM and Lab4b CM also had the ability to attenuate rotenone-induced apoptosis and necrosis with Lab4b inducing the greater effect. Both consortia showed an analogous ability to attenuate intracellular reactive oxygen species accumulation in SH-SY5Y cells although the differential upregulation of genes encoding glutathione reductase and superoxide dismutase by Lab4 CM and Lab4b CM, respectively, implicates the involvement of consortia-specific antioxidative mechanisms of action. This study implicates Lab4 and Lab4b as potential neuroprotective agents and justifies their inclusion in further in vivo studies.
\end{abstract}

Keywords: neurodegeneration, anti-oxidant, neurone, rotenone, in vitro

\section{Introduction}

Aging effects the physiology of every cell of the human body leading to changes and/or loss of function. The brain is particularly susceptible to deterioration and neurodegeneration; the progressive loss of structure or function of neurones, can be found to varying degrees in the brains of all age groups (Wyss-Coray, 2016). Such changes are irreversible, non-curative, contribute to agerelated declines in cognitive function and memory and are a major risk factor for neurological disorders, including Parkinson's and Alzheimer's disease (Wahl et al., 2017; Wyss-Coray, 2016). As the age of the population increases, so does the socio-economic burden of neurodegeneration thus highlighting the need to identify neuroprotective agents (Gillette-Guyonnet et al., 2013; Lalkovičová and Danielisová, 2016; Wahl et al., 2017; Wyss-Coray, 2016).
The mechanisms driving neurodegeneration are complicated and not fully understood although it is widely accepted that disturbances in hormonal balance, inflammation, metabolism and oxidative stress contribute. The relationship between the gut microbiota and the brain (the gut-brain-axis) and neurodegeneration is related to changes/imbalances in the composition of the gut microbiota causing decreases in population diversity and functionality (Dinan and Cryan, 2017). The gut-brain axis is a dynamic bi-directional system consisting of neurological, endocrine and immunological elements. If the microbial balance of the gut is disrupted, communication with the brain is disturbed and this has been associated with low grade inflammation, increased oxidative status, unbalanced energy homeostasis and increased cellular degeneration (Westfall et al., 2017). Alterations of the gut microbiota composition can affect many brain biological processes (e.g. development and neurogenesis) and behavioural traits (e.g. 
anxiety, depression, learning and memory). Probiotics are defined as 'live microorganisms which when administered in adequate amounts confer a health benefit on the host' (FAO/WHO, 2006) and have been demonstrated to play a role in maintaining microbial homeostasis within the gut (Kumar et al., 2016; Umbrello and Esposito, 2016). Indeed, probiotic organisms have shown beneficial effects on neurodegenerative disorders (Akbari et al., 2016; Bonfili et al., 2017, 2018; Kobayashi et al., 2017; Nimgampalle and Kuna, 2017) and animal studies with probiotics have shown clear neuroprotective effects (Ait-Belgnaoui et al., 2014; Distrutti et al., 2014; Möhle et al., 2016; Park et al., 2017). The aetiology of neurodegeneration is complex and the functional diversity of probiotics may facilitate the simultaneous targeting of multiple disease-related mechanisms (Elufioye et al., 2017; Faden and Stoica, 2007). Of particular interest are the antioxidant properties of such bacteria (Mishra et al., 2015; Wang et al., 2017) that can target the susceptibility of neurones to oxidative damage, a key driver of neurodegeneration (Liu et al., 2017).

The Lab4 and Lab4b probiotic consortia comprise distinct populations of Bifidobacterium spp. and Lactobacillus spp. Supplementation with Lab4b has been shown to reduce the incidence of allergy in infants (Allen et al., 2010, 2014) whilst Lab4 impacted on both inflammation (Davies et al., 2018; Garaiova et al., 2015; Hepburn et al., 2013) and metabolism (Michael et al., 2016, 2017). Some probiotic bacteria are known producers of metabolites with neuromodulatory capability (Sarkar et al., 2016) and Lab4 has been shown to improve memory and alter brain metabolite profiles in aging rats (O'Hagan et al., 2017). The aim of this in vitro study was to compare the neuroprotective potential of two distinct microbial populations (Lab4 and Lab4b) by assessing their ability to protect SH-SY5Y neuronal cells against oxidative stress and investigate the potential modes of action of the two different consortia.

\section{Methods and reagents}

\section{Materials and reagents}

All materials and reagents were purchased from SigmaAldrich (Poole, UK) unless otherwise stated.

\section{Preparation of bacterial cultures}

DeMan Rogosa Sharpe (MRS) broth $(10 \mathrm{ml})$ was inoculated with freeze dried powder (10 mg) of Lab4 (composed of Lactobacillus acidophilus CUL21 (NCIMB 30156), L. acidophilus CUL60 (NCIMB 30157), Bifidobacterium bifidum CUL20 (NCIMB 30153) and Bifidobacterium animalis subsp. lactis CUL34 (NCIMB 30172)) or Lab4b (composed of Lactobacillus salivarius CUL61 (NCIMB 30211), Lactobacillus paracasei CUL08 (NCIMB 30154), Bifidobacterium bifidum CUL20 (NCIMB 30153), B. animalis subsp. lactis CUL34 (NCIMB 30172)) and incubated anaerobically ( $10 \%$ carbon dioxide, $10 \%$ hydrogen and $80 \%$ nitrogen) without shaking at $37^{\circ} \mathrm{C}$ for $18 \mathrm{~h}$.

\section{Preparation of Lab4 and Lab4b conditioned media}

Bacteria were harvested from $18 \mathrm{~h}$ cultures by centrifugation $(2,500 \times g$ for $20 \mathrm{~min})$, suspended in phosphate buffered saline (PBS), and centrifuged (2,500 $\times g$ for $20 \mathrm{~min})$. The resulting bacterial pellet was adjusted to a concentration of $1 \times 10^{9} \mathrm{cfu} / \mathrm{ml}$ in a $1: 1 \mathrm{mix}$ of Dulbecco's Modified Eagle's medium and Ham F-12 medium (DMEM/F12) and incubated under anaerobic conditions at $37^{\circ} \mathrm{C}$ for 5 $\mathrm{h}$, centrifuged $(2,500 \times g$ for $20 \mathrm{~min})$ and the supernatant passed through a $0.22 \mu \mathrm{m}$ filter (Gilson, Bedfordshire, UK) to produce the conditioned media (CM) preparation. The CM were adjusted to $\mathrm{pH} 7.4 \mathrm{using} 1 \mathrm{M} \mathrm{NaOH}$ and supplemented with $100 \mathrm{U} / \mathrm{ml}$ penicillin and $100 \mathrm{U} / \mathrm{ml}$ streptomycin (Labtech, Heathfield, UK). Enumeration at the start and end of the $5 \mathrm{~h}$ incubation period confirmed that no change in bacterial number occurred during this period (data not shown). DMEM/F12 supplemented with $100 \mathrm{U} / \mathrm{ml}$ penicillin and $100 \mathrm{U} / \mathrm{ml}$ streptomycin was used as a diluent for the preparation of CM doses and as a control for CM.

\section{Total antioxidant capacity}

The total antioxidant capacity (TAC) of intact cells and CM was determined using the Total Antioxidant Capacity Assay Kit (Abcam, Cambridge, UK) in accordance with the manufacturer's instructions. Prior to measurement, intact cells were washed twice in phosphate buffered saline (PBS) to remove all traces of culture media and the TAC value for PBS subtracted from experimental values. The TAC of serum-free DMEM/F12 was subtracted from CM values. Data are presented as the mean Trolox equivalent antioxidant capacity (mM).

\section{SH-SY5Y cell culture, differentiation and stimulation}

SH-SY5Y cells were maintained in DMEM/F12 (Labtech) supplemented with $10 \%(\mathrm{v} / \mathrm{v})$ heat inactivated foetal bovine serum (Labtech), penicillin $(100 \mathrm{U} / \mathrm{ml})$ and streptomycin $(100 \mathrm{U} / \mathrm{ml})$ at $37{ }^{\circ} \mathrm{C}$ in $5 \% \mathrm{CO}_{2}$ and $95 \%$ humidity. Approximately $24 \mathrm{~h}$ before experimentation, cells of $\sim 80 \%$ confluency were seeded at $5 \times 10^{5}$ cells $/ \mathrm{cm}^{2}$ into appropriate tissue culture plates (Costar, Cambridge, UK). Cells between passage 21 and 30 were used in this study. Where differentiation was required, SH-SY5Y were incubated with DMEM/F12 supplemented with $1 \%(\mathrm{v} / \mathrm{v})$ heat inactivated foetal bovine serum, penicillin $(100 \mathrm{U} / \mathrm{ml})$, streptomycin $(100 \mathrm{U} / \mathrm{ml})$ and $10 \mu \mathrm{M}$ all-trans retinoic acid (ATRA) for 5 days. Differentiating cells were incubated under light-free conditions and the media changed every 2 days. Rotenone was dissolved in dimethyl sulfoxide (DMSO) and applied 
to SH-SY5Y cells at a concentration of $250 \mathrm{nM}$ for viability and apoptosis/necrosis assays (Supplementary Figure $\mathrm{S} 1 \mathrm{~A}$ ) or $10 \mu \mathrm{M}$ for intracellular reactive oxygen species (ROS) assays (Supplementary Figure S1B). DMSO was also included at the relevant concentration in the controls for these experiments and is referred to as the vehicle control. 1-Methyl-4-phenylpyridinium iodide ( $\mathrm{MPP}+$ ) was dissolved in water that was included at the relevant concentration in the controls for these experiments and is referred to as the vehicle control.

\section{MTT-assay}

Following exposure to experimental conditions, SH-SY5Y cells were washed with $200 \mu$ l of warm PBS (pH 7.4) before incubation with $100 \mu \mathrm{l}$ of 3-(4,5-dimethythiazol-2-yl)-2,5diphenyl tetrazolium bromide (MTT) solution $(500 \mu \mathrm{g} / \mathrm{ml}$ in DMEM/F12) for $2 \mathrm{~h}$ in $5 \% \mathrm{CO}_{2}$ and $95 \%$ humidity. The cells were then washed in $200 \mu \mathrm{l}$ of PBS $\left(\mathrm{pH} 7.4,37^{\circ} \mathrm{C}\right.$ ) before the addition of $100 \mu \mathrm{l}$ of DMSO. The absorbance at 570 $\mathrm{nm}$ was read using a colorimetric spectrophotometer and viability was expressed as percentage survival compared to untreated cells or cells treated with the appropriate vehicle that have been arbitrarily assigned as $100 \%$.

\section{Crystal violet assay}

Following exposure to experimental conditions, SH-SY5Y cells were washed with $200 \mu \mathrm{l}$ of PBS $\left(\mathrm{pH} 7.4,37^{\circ} \mathrm{C}\right.$ ) before incubation with $0.2 \%(\mathrm{w} / \mathrm{v})$ crystal violet solution (in $10 \%$ $(\mathrm{v} / \mathrm{v})$ ethanol) for $5 \mathrm{~min}$ at room temperature. The cells

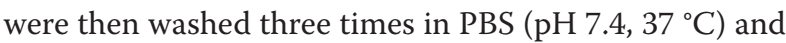
left to dry at room temperature overnight before $150 \mu \mathrm{l}$ of $10 \%$ acetic acid solution $(\mathrm{v} / \mathrm{v})$ was added to each well and the absorbance read at $595 \mathrm{~nm}$ using a colorimetric spectrophotometer. Viability is expressed as percentage survival compared to untreated cells or cells treated with the appropriate vehicle that have been arbitrarily assigned as $100 \%$.

\section{Apoptosis and necrosis assay}

Levels of apoptosis and necrosis were measured in $\mathrm{SH}$ SY5Y cells exposed to experimental conditions using the RealTime-Glo ${ }^{\mathrm{Tm}}$ Annexin V Apoptosis and Necrosis Assay (Promega, Madison, WI, USA) in accordance with the manufacturer's instructions.

\section{Intracellular reactive oxygen species assay}

Intracellular ROS levels were measured in SH-SY5Y cells exposed to experimental conditions using the DCFDA Cellular ROS Detection Assay Kit (Abcam) in accordance with the manufacturer's instructions. SH-SY5Y cells were cultured in phenol red-free DMEM/F12 (Gibco, Paisley, UK) to eliminate assay interference by phenol red. Intracellular
ROS is expressed as a percentage compared to cells treated with the appropriate vehicle that have been arbitrarily assigned as $100 \%$.

\section{RT-qPCR}

Total RNA was isolated using Ribozol (Amresco LLC, Solon, $\mathrm{OH}, \mathrm{USA}$ ) and cDNA generated using the High Capacity cDNA Reverse Transcription Kit (Life Technologies, Paisley, UK) in accordance to the manufacturer's instructions. Real-time qPCR amplification was performed as previously described (Michael et al., 2016) using the oligonucleotide primers shown in Supplementary Table S1. Ratios of gene expression compared to untreated cells were determined using $2^{-(\Delta C t 1-\Delta C t 2)}$, where $\Delta C t$ represents the difference between the threshold cycle (CT) for each target gene and glyceraldehyde 3-phosphate dehydrogenase (GAPDH) or $\beta$-actin mRNA transcript levels.

\section{Statistical analysis}

All data are presented as the mean \pm standard error of the mean (SEM) of the assigned number of independent experiments. The normality of all data was assessed using histograms and Q-Q plots and data transformations were applied where appropriate. For single comparisons, values of $P$ were determined using a Student's $t$-test. For multiple comparisons, $P$-values were determined using one-way analysis of variance (ANOVA) with Tukey's or Dunnett's T3 post-hoc analysis or, where the data was not normally distributed, Kruskal-Wallis ANOVA with Dunns post-hoc analysis. All statistical tests were performed using SPSS statistical software package version 22 (IBM, Armonk, NY, USA). Differences were considered significant when $P<0.05$.

\section{Results}

\section{Lab4 and Lab4b consortia possess antioxidant capacity}

Antioxidant activity was observed in three independent cultures of whole cells of the Lab4 and Lab4b consortia $(0.105 \pm 0.010$ and $0.233 \pm 0.023 \mathrm{mM}$, respectively) and CM preparations $(0.735 \pm 0.052$ and $0.084 \pm 0.057 \mathrm{mM}$, respectively). Significant differences were observed in whole cell preparations of Lab4 compared to Lab4b $(P=0.042)$ and in CM from Lab4 compared to Lab4b $(P=0.026)$.

\section{Lab4 CM and Lab4b CM can impact upon the viability of undifferentiated SH-SY5Y cells}

Undifferentiated SH-SY5Y cells were exposed to various doses of Lab4 and Lab4b CM for $48 \mathrm{~h}$ and viability was assessed using the MTT assay (Figure 1A). There was a trend of improved viability at all doses that reached significance for the $10 \%$ Lab4 CM dose $(14.8 \%, P=0.031)$ compared to the control. Similar results were obtained using the $\mathrm{CV}$ 

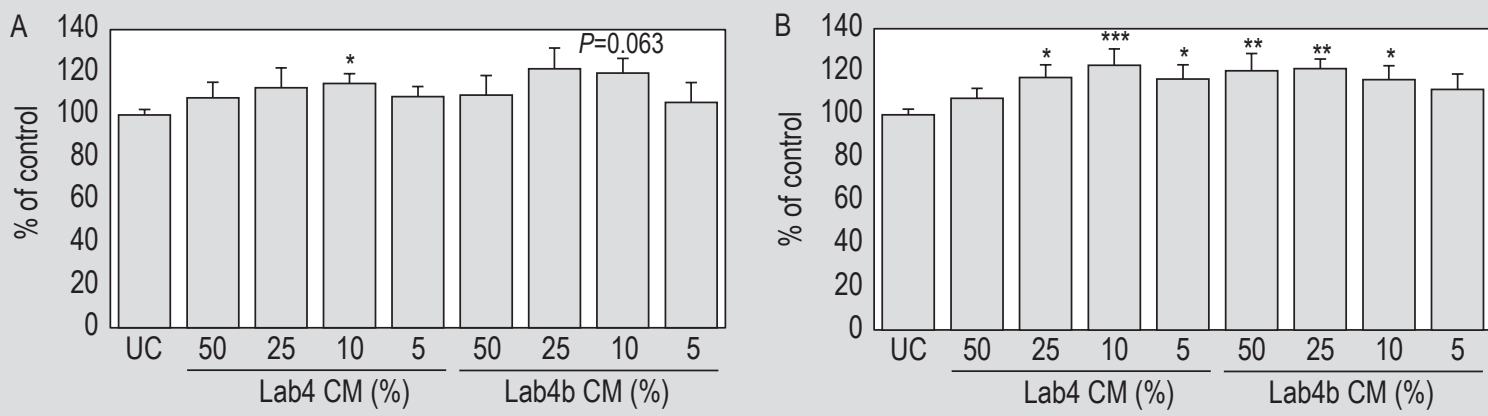

Figure 1. The effect of Lab4 and Lab4b CM on the viability of undifferentiated SH-SY5Y cells. The viability of undifferentiated SHSY5Y cells that were treated with either serum-free DMEM/F12 (untreated control, UC) or treated with various doses of Lab4 or Lab4b CM for $48 \mathrm{~h}$ was assessed by (A) MTT assay or (B) Crystal Violet assay. Data are expressed as a percentage of the control that has been arbitrarily assigned as $100 \%$. The data are presented as the mean \pm standard error of the mean of four independent experiments. ${ }^{*} P<0.05,{ }^{* *} P<0.01,{ }^{* * *} P<0.001$ or as stated vs untreated cells.

assay (Figure 1B) where significant improvements were observed for $25 \%$ (17.4\%, $P=0.018), 10 \%(23.1 \%, P<0.001)$ and $5 \%(16.6 \%, P=0.031) \mathrm{CM}$ from Lab4 and 50\% (20.5\%, $P=0.002), 25 \%(21.4 \%, P=0.001)$ and $10 \%(16.4 \%, P=0.034)$ CM from Lab4b compared to the control. No statistically significant differences were observed between doses or between Lab4 and Lab4b in either assay.

\section{Lab4 CM and Lab4b CM protect undifferentiated SH-SY5Y cells against rotenone-induced loss of viability}

The toxicity of doses of rotenone in undifferentiated $\mathrm{SH}$ SY5Y cells was measured (Supplementary Figure S1A). Survival rates in SH-SY5Y cells exposed to $250 \mathrm{nM}$ rotenone were $53.4 \%(P<0.001)$ of the control (Figure $2 \mathrm{~A})$ and survival rates in the presence of rotenone were improved up to $72.1 \%(P=0.006)$ and $67.0 \%(P=0.130)$ with Lab4 and Lab4b CM, respectively (see Supplementary Figure S2 for optimisation experiments). This neuroprotective capability was confirmed in $\mathrm{CV}$ assays (Figure 2B) where the survival rate of $75.9 \%(P<0.001)$ in rotenone exposed cells increased to $91.9 \%(P=0.062)$ in the presence of Lab4 CM and $96.9 \%$ $(P=0.007)$ in the presence of Lab4b CM. No statistically significant differences were observed between Lab4 and Lab4b in these experiments.

\section{Pre-incubation with Lab4 CM or Lab4b CM protects undifferentiated SH-SY5Y cells against rotenone-induced loss of viability}

Undifferentiated SH-SY5Y cells were incubated with Lab4 or Lab4b (50\% CM) for $2 \mathrm{~h}$ prior to the exposure of the neuronal cells to $250 \mathrm{nM}$ rotenone. A survival rate of $70.9 \%$ $(P<0.001)$ was observed in cells treated with rotenone and this increased to $86.7 \%(P=0.053)$ in Lab4 and $90.3 \%$ $(P=0.033)$ in Lab4b pre-treated cells (Figure $2 \mathrm{C})$. These findings were corroborated using $\mathrm{CV}$ assays (Figure 2D) where the neuronal survival rate increased from $56.2 \%$
$(P<0.001)$ in rotenone-treated cells to $69.4 \%(P=0.035)$ and $62.5 \%(P=0.523)$ in the Lab4 and Lab4b pre-treated cells, respectively. No statistically significant differences were observed between Lab4 and Lab4b in these experiments.

\section{Protection of ATRA-differentiated SH-SY5Y cells against rotenone-induced loss of viability}

SH-SY5Y cell were incubated with $10 \mu \mathrm{M}$ ATRA for 5 days to induce differentiation as described elsewhere (Kovalevich and Langford, 2013) and changes to cell morphology and gene expression levels of tropomyosin receptor kinase B (Kaplan et al., 1993) confirmed the responsiveness of SH-SY5Y cells to these conditions (Supplementary Figure S4). In ATRA-treated SH-SY5Y cells (Figure 2E), 84.8\% $(P<0.001)$ of cells remained viable after rotenone exposure compared to the control and the survival rate increased to $104.9 \%(P<0.001)$ and $98.5 \%(P=0.029)$ as a result of co-incubation with Lab4 and Lab4b CM respectively. Pre-incubation for $2 \mathrm{~h}$ with Lab4 and Lab4b CM prior to rotenone challenge (Figure $2 \mathrm{~F}$ ) significantly improved the survival rate from $86.2 \%(P<0.001)$ in rotenone-treated cells to $109.3 \%(P<0.001)$ and $107.2 \%(P<0.001)$, respectively. These improvements in survival rate were also significantly different compared to the control $(P<0.001$ and $P=0.013$ respectively). No statistically significant differences were observed between Lab4 and Lab4b in these experiments.

\section{Lab4 CM and Lab4b CM protect undifferentiated SH-SY5Y cells against MPP+ induced loss of viability}

The toxicity of doses of MPP+ in undifferentiated SHSY5Y cells was measured (Supplementary Figure S3) and exposure to $2 \mu \mathrm{M} \mathrm{MPP}+$ reduced viability to $62.4 \%$ $(P<0.001)$ of the control in these cells (Figure 3A). In the presence of CM from Lab4 and Lab4 there was indication of improved survival rates at all concentrations tested compared to rotenone-treated cells although statistical 

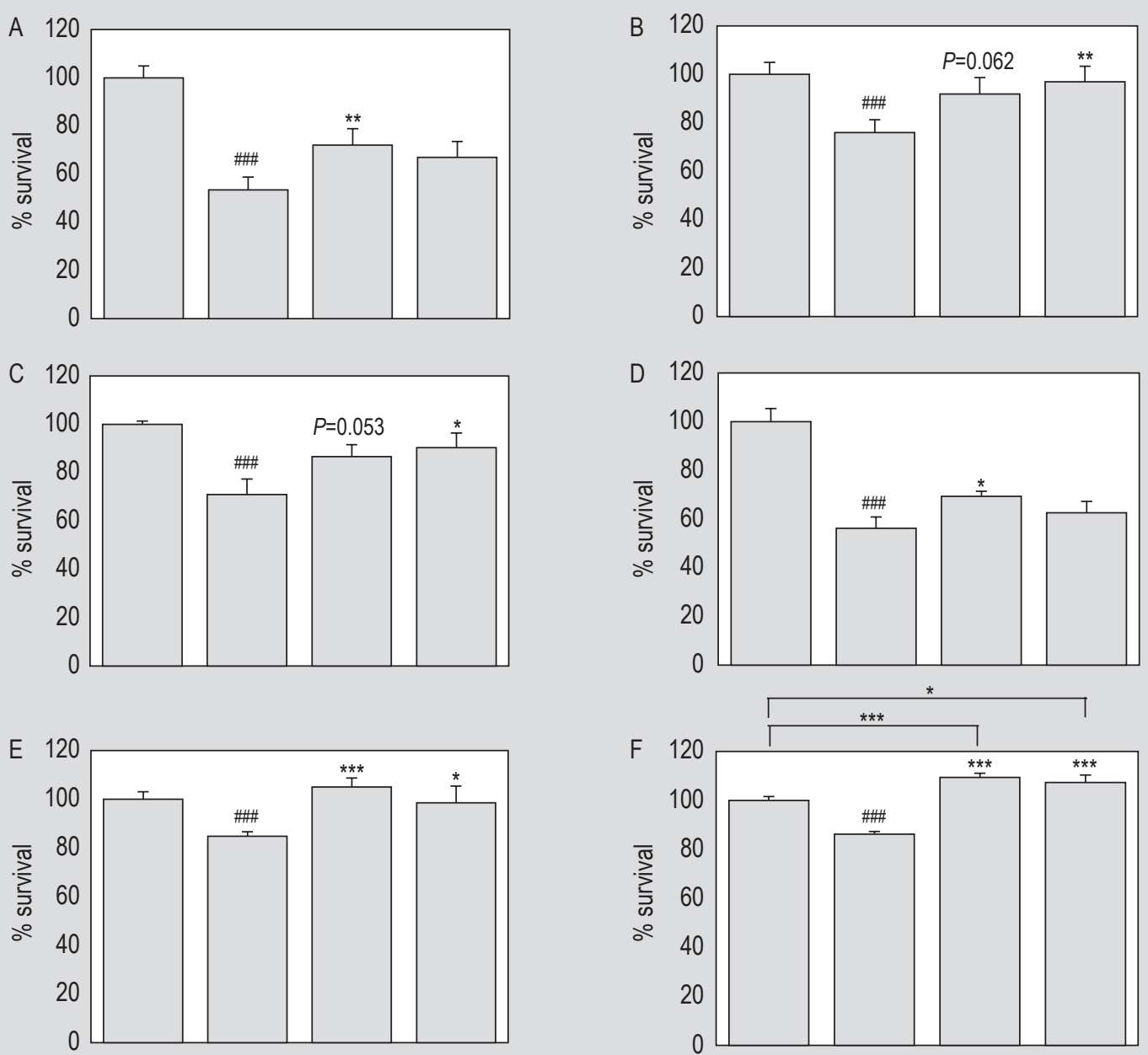

Vehicle control $\quad+$

Rotenone (250 nM)

Lab4 CM (\%)

Lab4b CM $(\%)$

50

50

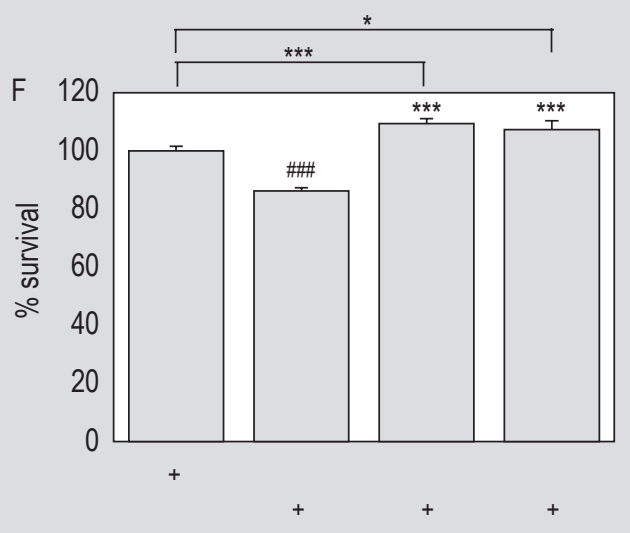

50

50

Figure 2. The effect of Lab4 and Lab4b CM on rotenone-induced toxicity in undifferentiated and ATRA-differentiated SH-SY5Y cells. The viability of undifferentiated SH-SY5Y cells that were treated with the vehicle control (DMSO) or $250 \mathrm{nM}$ rotenone or $250 \mathrm{nM}$ rotenone with Lab4 or Lab4b CM for $24 \mathrm{~h}$ was assessed by (A) MTT assay or (B) Crystal Violet assay. The viability of undifferentiated SH-SY5Y cells that were pre-incubated with serum-free DMEM/F12 or CM from Lab4 or Lab4b for $2 \mathrm{~h}$ prior to the addition of the vehicle control (DMSO) or $250 \mathrm{nM}$ rotenone for $24 \mathrm{~h}$ was assessed by (C) MTT assay or (D) Crystal Violet assay. The viability of differentiated SH-SY5Y cells (10 $\mu \mathrm{M}$ ATRA and 1\% FCS for 5 days) that were treated with (E) vehicle control (DMSO) or $250 \mathrm{nM}$ rotenone or $250 \mathrm{nM}$ rotenone with Lab4 or Lab4b CM or (F) pre-incubated with serum-free DMEM/F12 or $250 \mathrm{nM}$ rotenone for $24 \mathrm{~h}$ was assessed by MTT assay. Data are expressed as percentage survival compared to the vehicle control that has been arbitrarily assigned as $100 \%$. The data are presented as the mean \pm standard error of the mean of at least three independent experiments. ${ }^{*} P<0.05,{ }^{* *} P<0.01,{ }^{* * *} P<0.001$ or as stated vs the rotenone-treated cells or ${ }^{\# \#} P<0.001$ vs the vehicle control.

significance was not achieved. In CV assays, exposure to $2 \mu \mathrm{M} \mathrm{MPP}+$ reduced viability to $82.6 \%(P<0.001)$ of the control (Figure $3 \mathrm{~B}$ ) and indication of improved survival was observed upon the addition of Lab4 and Lab4b at all concentrations tested that, in the case of 25 and 50\% CM from Lab4b, were significantly improved to $108.2 \%$ $(P=0.033)$ and $99.9 \%(P=0.010)$, respectively. No statistically significant differences were observed between Lab4 and Lab4b in these experiments.
Lab4 and Lab4b CM protect undifferentiated SH-SY5Y cells against rotenone-induced apoptosis and necrosis

Exposure of undifferentiated SH-SY5Y cells to $250 \mathrm{nM}$ rotenone for $24 \mathrm{~h}$ resulted in a significant 1.74-fold $(P<0.001)$ increase in apoptosis compared to the untreated control (Figure 4A) and in the presence of Lab4 and Lab4b CM, reduced apoptotic activity was observed (1.54-fold, $P=0.040$ and 1.18-fold, $P<0.001$ respectively). After $48 \mathrm{~h}$ exposure (Figure 4B), the levels of apoptosis were lower but similar 
A

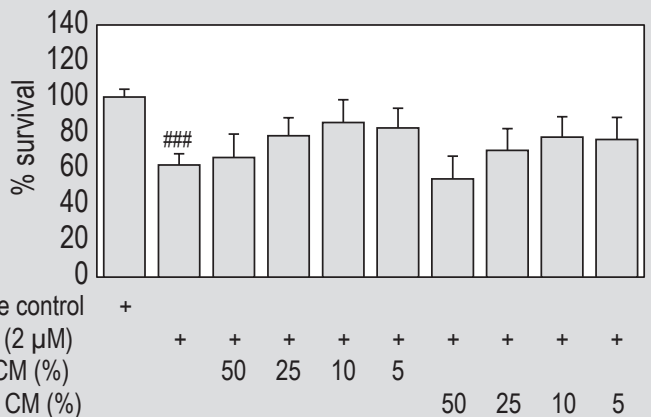

B

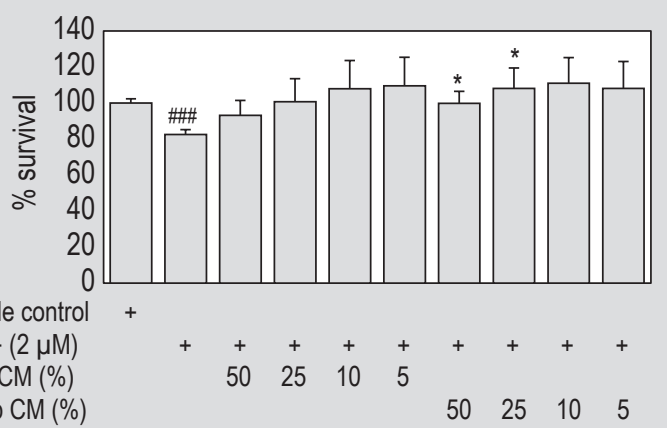

Figure 3. The effect of Lab4 and Lab4b CM on 1-methyl-4-phenylpyridinium iodide (MPP+)-induced toxicity in undifferentiated SH-SY5Y cells. The viability of undifferentiated SH-SY5Y cells that were treated with the vehicle control (water) or $2 \mu M$ MPP+ or 2 $\mu M$ MPP+ plus the stated concentration of Lab4 or Lab4b CM for $24 \mathrm{~h}$ was assessed by (A) MTT assay or (B) Crystal Violet assay. Data are expressed as percentage survival compared to the vehicle control that has been arbitrarily assigned as $100 \%$. The data are presented as the mean \pm standard error of the mean of at least five independent experiments. ${ }^{*} P<0.05$ vs the MPP+-treated cells or $P<0.001$ vs the vehicle control.

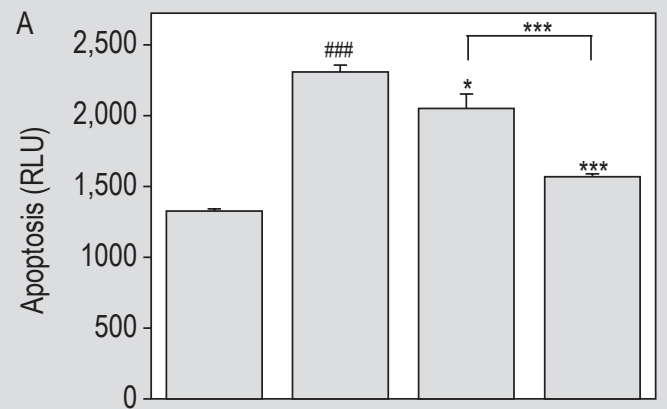

C

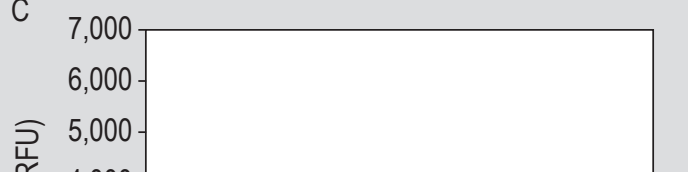

$\frac{1}{\alpha}$
$\frac{1}{0}$
$\frac{0}{d}$
$\frac{d}{2}$

$\begin{array}{ll}\frac{\text { D }}{\text { W }} & 4,000 \\ \text { 은 } & 3,000 \\ \text { d } & 2,000\end{array}$

$\stackrel{ \pm}{z} 2,000$

1000

0

Vehicle control

Rotenone $(250 \mathrm{nM})$

Lab4 CM (\%)

Lab4b CM (\%)

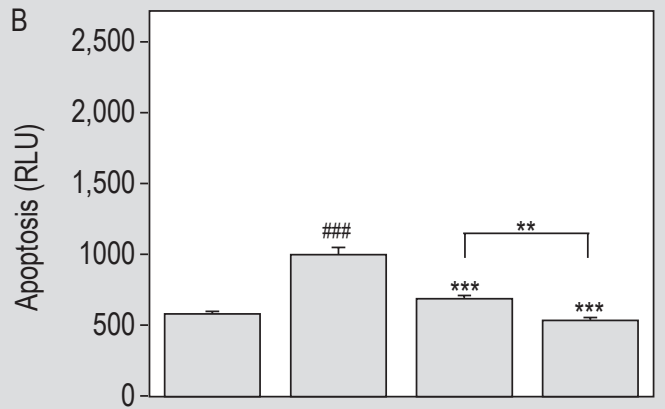

D

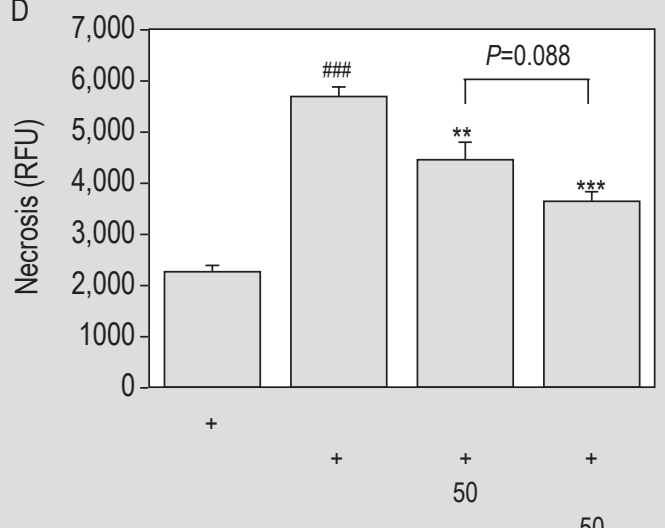

Figure 4. The effect of Lab4 and Lab4b CM on rotenone-induced apoptosis and necrosis in undifferentiated SH-SY5Y cells. Levels of apoptosis $(\mathrm{A}+\mathrm{B})$ or necrosis $(\mathrm{C}+\mathrm{D})$ were assessed in undifferentiated $\mathrm{SH}-\mathrm{SY} 5 \mathrm{Y}$ cells that were treated with the vehicle control (DMSO) or $250 \mathrm{nM}$ rotenone or $250 \mathrm{nM}$ rotenone with Lab4 or Lab4b CM for $24 \mathrm{~h}(\mathrm{~A}+\mathrm{C})$ or $48 \mathrm{~h}(\mathrm{C}+\mathrm{D})$. The data are presented as the mean \pm standard error of the mean of relative luminescent units $(R L U, A+B)$ or relative fluorescent units $(R F U, C+D)$ from five independent experiments. ${ }^{*} P<0.05,{ }^{* *} P<0.01$ and ${ }^{* * *} P<0.001$ vs rotenone-treated cells or ${ }^{* \#} P<0.001$ vs the vehicle control.

patterns of change were observed with rotenone inducing a 1.71 -fold increase $(P<0.001)$ that was reduced to 1.18 -fold $(P<0.001)$ and 0.92 -fold $(P<0.001)$ in the presence of Lab4 and Lab4b CM, respectively. Reductions in apoptosis were significantly greater with Lab4b compared to Lab4 at both $24 \mathrm{~h}(P<0.001$, Figure $4 \mathrm{~A})$ and $48 \mathrm{~h}(P=0.001$, Figure $4 \mathrm{~B})$. 
Apoptosis precedes secondary necrosis and at $24 \mathrm{~h}$ low levels of necrosis were detected (Figure 4C) with a trend towards increased levels of necrosis in rotenone-treated cells when compared to the control (1.64-fold, $P=0.055$, Figure $4 \mathrm{C}$ ) that remained unchanged in the presence of Lab4 and Lab4b. Higher levels of necrosis were detected at $48 \mathrm{~h}$ and rotenone exposure induced a significant increase compared to the control (2.52-fold, $P<0.001$, Figure 4D). In the presence of Lab4 or Lab4b necrosis rates were 1.97fold $(P=0.006)$ and 1.60 -fold $(P<0.001)$, respectively, in line with the reduced apoptotic activity. Lab4b showed a trend towards increased capability compared to Lab4 ( $P=0.088$, Figure 4D) similar to the effects observed during apoptosis.

\section{Lab4 and Lab4b CM protect undifferentiated cells against ROS and induce genes involved in antioxidant defence}

Exposure of undifferentiated SY-SY5Y cells to $10 \mu \mathrm{M}$ rotenone for $6 \mathrm{~h}$ increased cellular ROS levels by 1.43fold $(P<0.001)$ compared to the control and the degree of this effect was significantly reduced to 1.14 -fold $(P<0.001)$ in the presence of Lab4 CM and 1.23-fold $(P=0.002)$ in the presence of Lab4b CM (Figure 5). No significant differences were observed between Lab4 and Lab4b. At rotenone concentrations between $250 \mathrm{nM}$ and $10 \mu \mathrm{M}$, no increase in intracellular ROS levels was detected (Supplementary Figure S1B).

The expression of key genes involved in endogenous antioxidant defence in undifferentiated SH-SY5Y cells exposed to Lab4 and Lab4b for $24 \mathrm{~h}$ can be seen in Table

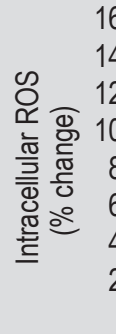

Vehicle control

Rotenone $(10 \mu \mathrm{M})$

Lab4 CM (\%)

Lab4b CM (\%)

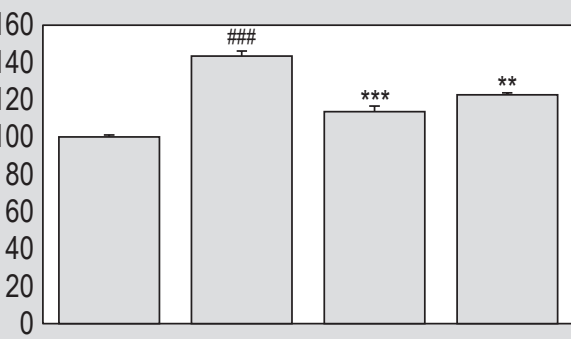

$+$
+
50
Figure 5. The effect of Lab4 and Lab4b CM on rotenone-induced cellular ROS accumulation in undifferentiated SH-SY5Y cells. Intracellular ROS levels in undifferentiated SH-SY5Y cells that were treated with the vehicle control (DMSO) or $10 \mu \mathrm{M}$ rotenone or $10 \mu \mathrm{M}$ rotenone with Lab4 or Lab4b CM for $6 \mathrm{~h}$. Data are expressed as a percentage compared to the vehicle control that has been arbitrarily assigned as $100 \%$. The data is presented as the mean \pm standard error of the mean of four independent experiments. ${ }^{* *} P<0.01$, ${ }^{* *} P<0.001$ vs rotenone-treated cells or ${ }^{\# \#} P<0.001$ vs the vehicle control.
1 and demonstrates a significant 1.51 -fold $(P=0.022)$ induction of glutathione reductase (GSR) expression with Lab4 and an 18.12-fold $(P<0.001)$ induction in superoxide dismutase 2 (SOD2) expression in those treated with Lab4b. No changes in expression were observed for superoxide dismutase 1 (SOD1), glutathione peroxidase (GPx1) or catalase nor any differences between Lab4 and Lab4b for any gene.

\section{Discussion}

In an SH-SY5Y neuronal model, both Lab4 and Lab4b populations of microorganisms induced neuroprotective effects on challenged cells. Both consortia were found to possess antioxidant activity and were able to induce proliferation in SH-SY5Y cells. When either undifferentiated or retinoic acid-differentiated SH-SY5Y cells were challenged with the neurotoxin, rotenone, pre- or coexposure of the cells to Lab4 or Lab4b partly prevented rotenone-induced loss of viability. There were also protective effects against the development of apoptosis and necrosis and the intracellular accumulation of ROS.

Neurodegeneration occurs as a result of oxidative damage (Westfall et al., 2017) and there is a growing interest in the role of probiotic bacteria with the ability to impact upon the antioxidant capacity of the host (Wang et al., 2017). Improvements in oxidative status alongside improved behaviour have been observed in an Alzheimer's disease mouse model supplemented with a multi-strain probiotic composed of strains of Bifidobacterium and Lactobacillus (Bonfili et al., 2017, 2018). Probiotics are thought to impart antioxidant effects by modulating endogenous antioxidant defence mechanisms and/or via the secretion of metabolites with antioxidant properties (Wang et al., 2017). Antioxidant activities were detected in both viable cells and conditioned media of Lab4 and Lab4b, albeit at differing levels, and

Table 1. Changes in gene expression of key antioxidant genes in undifferentiated SH-SY5Y cells exposed to CM from Lab4 and Lab4b. ${ }^{1}$

$\begin{array}{lll}\text { Gene } & \text { Lab4 } & \text { Lab4b } \\ \text { Superoxide dismutase 1 } & 1.19 \pm 0.40 & 0.85 \pm 0.21 \\ \text { Superoxide dismutase 2 } & 1.41 \pm 0.15 & 18.12 \pm 5.75^{\# \#} \\ \text { Glutathione peroxidase } & 1.06 \pm 0.15 & 0.80 \pm 0.12 \\ \text { Glutathione reductase } & 1.51 \pm 0.07^{\#} & 1.18 \pm 0.08 \\ \text { Catalase } & 1.09 \pm 0.11 & 0.80 \pm 0.17\end{array}$

${ }^{1}$ Gene expression is represented as a ratio in relation to an untreated control and is presented as the mean \pm standard error of the mean of three independent experiments. ${ }^{\#} P<0.05$ and ${ }^{\# \#} P<0.001$ vs the untreated control. 
highlighted both consortia as potential neuroprotective candidates.

Neurodegenerative disorders and age-related cognitive decline are associated with impaired neurogenesis; the process by which stem cells differentiate and proliferate into new neurones and other brain cells (Winner and Winkler, 2015). In our study, Lab4 and Lab4b induced proliferation in both undifferentiated SH-SY5Y cells (Figure 1) and rotenone-challenged ATRA differentiated SH-SY5Y cells (Figures 2F). The multi-strain probiotic, VSL\#3, has been shown to fully restore defective neurogenesis in antibiotictreated mice (Möhle et al., 2016) and a probiotic comprising Lactobacillus helveticus R0052 and Bifidobacterium longum R0175 supported mice exposed to chronic stress (Ait-Belgnaoui et al., 2014). Furthermore, other dietary antioxidants, such as the catechins found in green tea, have been shown to induce proliferation in undifferentiated SH-SY5Y cells and improve cognition in a murine model of brain aging (Pervin et al., 2017).

Neuronal cells are particularly vulnerable to oxidative damage (Lalkovičová and Danielisová, 2016) and both consortia possessed a comparable ability, as pre- and/or co-treatments, to attenuate losses in undifferentiated $\mathrm{SH}$ SY5Y cell viability in response to two well characterised neurotoxins and mediators of oxidative stress; rotenone and MPP+ (Giordano et al., 2012). The effects against rotenone are supported by the ability of both consortia to attenuate apoptosis and necrosis with Lab4b showing more activity than Lab4. These findings are consistent with an existing study showing the ability of glyceryl 1,3-dipalmitate, purified from the fermentation products of Lactobacillus paracasei subsp. paracasei NTU 101, to protect undifferentiated SH-SY5Y cells from oxygenglucose deprivation and reperfusion-induced oxidative stress (Cheng and Pan, 2017). Metabolites induced by the intestinal commensal, Ruminococcus albus, have also been shown to protect undifferentiated cells from hydrogen peroxide, MPP+ and sodium arsenate-induced oxidative damage (Park et al., 2017).

Previous probiotic studies in the SH-SY5Y model have been performed on undifferentiated (naïve) cells (Cheng and Pan, 2017; Park et al., 2017) and in the current study we have shown that the neuroprotective effects of the Lab4 and Lab4b consortia CM against the effects of rotenone, as pre- or co-incubations, are conserved in retinoic aciddifferentiated SH-SY5Y cells. Studies with differentiated SH-SY5Y cells are often favoured as these may represent a more relevant physiological format (Korecka et al., 2013; Lopes et al., 2010). It is worthy of note that Jantas et al. (2013) found that $10 \mu \mathrm{M}$ rotenone had little impact on the viability of undifferentiated cells that had been preexposed to reduced serum levels for $24 \mathrm{~h}$ whereas retinoic acid-differentiated cells were susceptible to rotenone under the same conditions (Jantas et al., 2013). This is in contrast to our study where the susceptibility of SH-SY5Y cells to rotenone was reduced upon retinoic acid-differentiation.

The mechanisms by which probiotic bacteria may impart neuroprotective effects have not been fully elucidated. Rotenone inhibits complex I of the mitochondrial electron transport chain leading to cellular ROS accumulation (Giordano et al., 2012) and our results indicate that Lab4 and Lab4b possess comparable abilities to attenuate ROS accumulation in SH-SY5Y cells. Cellular ROS levels are controlled, in part, by glutathione reductase, an enzyme that maintains intracellular pools of reduced glutathione (Espinosa-Diez et al., 2015), and expression levels of the encoding gene (GSR) were found to be elevated in the presence of Lab4. Another enzyme, manganese-dependant superoxide dismutase (MnSOD), also contributes to intracellular ROS homeostasis by converting superoxide into oxygen and hydrogen peroxide (Espinosa-Diez et al., 2015) and the expression of the encoding gene (SOD2) was induced in the presence of Lab4b. These data suggest the feasibility of different modes of action for Lab4 and Lab4b. These observations are supported by other studies showing reduced levels of ROS and increased levels of reduced glutathione and superoxide dismutase activity in the brains of Alzheimer's disease model mice supplemented with the VSL\#3 probiotic (Bonfili et al., 2018) and similar effects were seen with sodium arsenate-treated mice supplemented with Ruminococcus albus (Park et al., 2017). Future studies would investigate the ability of Lab4 and Lab4b CM to modulate the activity of complex I of the electron transport chain. 1-methylnicotinamde has been shown to impart neuroprotective effects by increasing complex I activity in rotenone-treated SH-SY5Y cells (Parsons et al., 2011).

Two potential limitations of our study involve firstly the duration of ATRA exposure used for the differentiation of SH-SY5Y cells. While there appears to be no consensus methodology for this process (Kovalevich and Langford, 2013; Xicoy et al., 2017), Lopes et al. (2010) required 7 days incubation to achieve a dopaminergic phenotype (Lopes et al., 2010) thus highlighting the possibility that 5 days incubation may be insufficient to reach terminal differentiation. Secondly, the metabolite profiles for the Lab4 and Lab4b consortia have not been determined. In the study of O'Hagan et al. (2017) rats supplemented daily with the Lab4 consortium from weaning through to sacrifice (18 months) had increased brain levels of lactate, myo-inositol, $\gamma$-aminobutyric acid, fumarate, inosine and alanine (O'Hagan et al., 2017) for which there is reported evidence of antioxidant activity (Chen et al., 2013; Grosser et al., 2004; Groussard et al., 2000; Gudkov et al., 2006; Jiang et al., 2011; Lin et al., 2016). Lactate is a major metabolic end-product of lactobacilli (Klein et al., 1998) which is able to pass through the blood-brain barrier (Proia et al., 2016). 
In summary, this in vitro study indicates that the conditioned media from the Lab4 and Lab4b probiotic consortia may have potential neuroprotective properties as demonstrated by the observed ability to protect SH-SY5Y cells against the actions of rotenone with distinct mechanisms of action attributed to each consortium. This study provides a basis for future targeted in vivo studies comparing the efficacy of these potentially beneficial probiotic consortia.

\section{Supplementary material}

Supplementary material can be found online at https://doi. org/10.3920/BM2018.0105.

Figure S1. The impact of rotenone on cell viability and intracellular ROS accumulation in undifferentiated $\mathrm{SH}$ SY5Y cells.

Figure S2. Lab4 and Lab4b exert a neuroprotective effect in rotenone-treated undifferentiated SH-SY5Y cells.

Figure S3. The impact of MPP+ on cell viability in undifferentiated SH-SY5Y cells.

Figure S4. Differentiation of SH-SY5Y cells with all-trans retinoic acid.

Table S1. Oligonucleotide primer sequences.

\section{Conflict of interest}

This study was supported by Cultech Ltd, Port Talbot, UK of which DRM, TSD, KEL, MDA and SFP are or were employees.

\section{References}

Ait-Belgnaoui, A., Colom, A., Braniste, V., Ramalho, L., Marrot, A., Cartier, C., Houdeau, E., Theodorou, V. and Tompkins, T., 2014. Probiotic gut effect prevents the chronic psychological stressinduced brain activity abnormality in mice. Neurogastroenterology and Motility 26: 510-520.

Akbari, E., Asemi, Z., Daneshvar Kakhaki, R., Bahmani, F., Kouchaki, E., Tamtaji, O.R., Hamidi, G.A. and Salami, M., 2016. Effect of probiotic supplementation on cognitive function and metabolic status in Alzheimer's disease: a randomized, double-blind and controlled trial. Frontiers in Aging Neuroscience 8: 256.

Allen, S.J., Jordan, S., Storey, M., Thornton, C.A., Gravenor, M., Garaiova, I., Plummer, S.F., Wang, D. and Morgan, G., 2010. Dietary supplementation with lactobacilli and bifidobacteria is well tolerated and not associated with adverse events during late pregnancy and early infancy. Journal of Nutrition 140: 483-488.

Allen, S.J., Jordan, S., Storey, M., Thornton, C.A., Gravenor, M.B., Garaiova, I., Plummer, S.F., Wang, D. and Morgan, G., 2014. Probiotics in the prevention of eczema: a randomised controlled trial. Archives of Disease in Childhood 99: 1014-1019.
Bonfili, L., Cecarini, V., Berardi, S., Scarpona, S., Suchodolski, J.S., Nasuti, C., Fiorini, D., Boarelli, M.C., Rossi, G. and Eleuteri, A.M., 2017. Microbiota modulation counteracts Alzheimer's disease progression influencing neuronal proteolysis and gut hormones plasma levels. Scientific Reports 7: 2426.

Bonfili, L., Cecarini, V., Cuccioloni, M., Angeletti, M., Berardi, S., Scarpona, S., Rossi, G. and Eleuteri, A.M., 2018. SLAB51 probiotic formulation activates SIRT1 pathway promoting antioxidant and neuroprotective effects in an AD mouse model. Molecular Neurobiology 55: 7987-8000.

Chen, Z., Tang, J., Sun, Y.Q. and Xie, J., 2013. Protective effect of $\gamma$-aminobutyric acid on antioxidation function in intestinal mucosa of Wenchang chicken induced by heat stress. Journal of Animal and Plant Sciences 23: 1634-1641.

Cheng, M.C. and Pan, T.M., 2017. Glyceryl 1,3-dipalmitate produced from Lactobacillus paracasei subspecies paracasei NTU 101 inhibits oxygen-glucose deprivation and reperfusion-induced oxidative stress via upregulation of peroxisome proliferator-activated receptor $\gamma$ in neuronal SH-SY5Y cells. Journal of Agricultural and Food Chemistry 65: 7926-7933.

Davies, T., Plummer, S., Jack, A., Allen, M. and Michael, D., 2018. Lactobacillus and Bifidobacterium promote antibacterial and antiviral immune response in human macrophages. Journal of Probiotics and Health 6: 195-202.

Dinan, T.G. and Cryan, J.F., 2017. Gut instincts: microbiota as a key regulator of brain development, ageing and neurodegeneration. Journal of Physiology 595: 489-503.

Distrutti, E., O’Reilly, J.A., McDonald, C., Cipriani, S., Renga, B., Lynch, M.A. and Fiorucci, S., 2014. Modulation of intestinal microbiota by the probiotic VSL\#3 resets brain gene expression and ameliorates the age-related deficit in LTP. PLoS ONE 9: e106503.

Elufioye, T.O., Berida, T.I. and Habtemariam, S., 2017. Plants-derived neuroprotective agents: cutting the cycle of cell death through multiple mechanisms. Evidence-Based Complementary and Alternative Medicine 2017: 3574012.

Espinosa-Diez, C., Miguel, V., Mennerich, D., Kietzmann, T., SánchezPérez, P., Cadenas, S. and Lamas, S., 2015. Antioxidant responses and cellular adjustments to oxidative stress. Redox Biology 6: 183-197.

Faden, A.I. and Stoica, B., 2007. Neuroprotection: challenges and opportunities. Archives of Neurology 64: 794-800.

Food and Agriculture Organization of the United Nations/World Health Organization (FAO/WHO), 2006. Probiotics in food. Health and nutritional properties and guidelines for evaluation. FAO Food and Nutritional paper no. 85. FAO, Rome, Italy. Available at: http:// tinyurl.com/8bccc3r.

Garaiova, I., Muchová, J., Nagyová, Z., Wang, D., Li, J.V., Országhová, Z., Michael, D.R., Plummer, S.F. and Ďuračková, Z., 2015. Probiotics and vitamin $C$ for the prevention of respiratory tract infections in children attending preschool: a randomised controlled pilot study. European Journal of Clinical Nutrition 69: 373-379.

Gillette-Guyonnet, S., Secher, M. and Vellas, B., 2013. Nutrition and neurodegeneration: epidemiological evidence and challenges for future research. British Journal of Clinical Pharmacology 75: 738755. 
Giordano, S., Lee, J., Darley-Usmar, V.M. and Zhang, J., 2012. Distinct effects of rotenone, 1-methyl-4-phenylpyridinium and 6-hydroxydopamine on cellular bioenergetics and cell death. PLoS ONE 7: e44610.

Grosser, N., Oberle, S., Berndt, G., Erdmann, K., Hemmerle, A. and Schröder, H., 2004. Antioxidant action of L-alanine: heme oxygenase- 1 and ferritin as possible mediators. Biochemical and Biophysical Research Communications 314: 351-355.

Groussard, C., Morel, I., Chevanne, M., Monnier, M., Cillard, J. and Delamarche, A., 2000. Free radical scavenging and antioxidant effects of lactate ion: an in vitro study. Journal of Applied Physiology 89: 169-175.

Gudkov, S.V., Shtarkman, I.N., Smirnova, V.S., Chernikov, A.V. and Bruskov, V.I., 2006. Guanosine and inosine as natural antioxidants and radioprotectors for mice exposed to lethal doses of gammaradiation. Doklady Biochemistry and Biophysics 407: 47-50.

Hepburn, N.J., Garaiova, I., Williams, E.A., Michael, D.R. and Plummer, S., 2013. Probiotic supplement consumption alters cytokine production from peripheral blood mononuclear cells: a preliminary study using healthy individuals. Beneficial Microbes 4: 313-317.

Jantas, D., Roman, A., Kuśmierczyk, J., Lorenc-Koci, E., Konieczny, J., Lenda, T. and Lasoń, W., 2013. The extent of neurodegeneration and neuroprotection in two chemical in vitro models related to Parkinson's disease is critically dependent on cell culture conditions. Neurotoxicity Research 24: 41-54.

Jiang, W.D., Wu, P., Kuang, S.Y., Liu, Y., Jiang, J., Hu, K., Li, S.H., Tang, L., Feng, L. and Zhou, X.Q., 2011. Myo-inositol prevents copperinduced oxidative damage and changes in antioxidant capacity in various organs and the enterocytes of juvenile Jian carp (Cyprinus carpio var. Jian). Aquatic Toxicology 105: 543-551.

Kaplan, D.R., Matsumoto, K., Lucarelli, E. and Thiele, C.J., 1993. Induction of TrkB by retinoic acid mediates biologic responsiveness to BDNF and differentiation of human neuroblastoma cells. Eukaryotic Signal Transduction Group. Neuron 11: 321-331.

Klein, G., Pack, A., Bonaparte, C. and Reuter, G., 1998. Taxonomy and physiology of probiotic lactic acid bacteria. International Journal of Food Microbiology 41: 103-125.

Kobayashi, Y., Sugahara, H., Shimada, K., Mitsuyama, E., Kuhara, T., Yasuoka, A., Kondo, T., Abe, K. and Xiao, J.Z., 2017. Therapeutic potential of Bifidobacterium breve strain A1 for preventing cognitive impairment in Alzheimer's disease. Scientific Reports 7: 13510.

Korecka, J.A., Van Kesteren, R.E., Blaas, E., Spitzer, S.O., Kamstra, J.H., Smit, A.B., Swaab, D.F., Verhaagen, J. and Bossers, K., 2013. Phenotypic characterization of retinoic acid differentiated SH-SY5Y cells by transcriptional profiling. PLoS ONE 8: e63862.

Kovalevich, J. and Langford, D., 2013. Considerations for the use of SHSY5Y neuroblastoma cells in neurobiology. Methods in Molecular Biology 1078: 9-21.

Kumar, M., Babaei, P., Ji, B. and Nielsen, J., 2016. Human gut microbiota and healthy aging: recent developments and future prospective. Nutrition and Healthy Aging 4: 3-16.

Lalkovičová, M. and Danielisová, V., 2016. Neuroprotection and antioxidants. Neural Regeneration Research 11: 865-874.
Lin, R., Cai, J., Kostuk, E.W., Rosenwasser, R. and Iacovitti, L., 2016. Fumarate modulates the immune/inflammatory response and rescues nerve cells and neurological function after stroke in rats. Journal of Neuroinflammation 13: 269.

Liu, Z., Zhou, T., Ziegler, A.C., Dimitrion, P. and Zuo, L., 2017. Oxidative stress in neurodegenerative diseases: from molecular mechanisms to clinical applications. Oxidative Medicine and Cellular Longevity 2017: 2525967.

Lopes, F.M., Schröder, R., Da Frota, M.L., Zanotto-Filho, A., Müller, C.B., Pires, A.S., Meurer, R.T., Colpo, G.D., Gelain, D.P., Kapczinski, F., Moreira, J.C., Fernandes, M.a.C. and Klamt, F., 2010. Comparison between proliferative and neuron-like SH-SY5Y cells as an in vitro model for Parkinson disease studies. Brain Research 1337: 85-94.

Michael, D.R., Davies, T.S., Moss, J.W.E., Calvente, D.L., Ramji, D.P., Marchesi, J.R., Pechlivanis, A., Plummer, S.F. and Hughes, T.R., 2017. The anti-cholesterolaemic effect of a consortium of probiotics: an acute study in C57BL/6J mice. Scientific Reports 7: 2883.

Michael, D.R., Moss, J.W., Calvente, D.L., Garaiova, I., Plummer, S.F. and Ramji, D.P., 2016. Lactobacillus plantarum CUL66 can impact cholesterol homeostasis in Caco-2 enterocytes. Beneficial Microbes 7: 443-451.

Mishra, V., Shah, C., Mokashe, N., Chavan, R., Yadav, H. and Prajapati, J., 2015. Probiotics as potential antioxidants: a systematic review. Journal of Agriculture and Food Chemistry 63: 3615-3626.

Möhle, L., Mattei, D., Heimesaat, M.M., Bereswill, S., Fischer, A., Alutis, M., French, T., Hambardzumyan, D., Matzinger, P., Dunay, I.R. and Wolf, S.A., 2016. Ly6C(hi) monocytes provide a link between antibiotic-induced changes in gut microbiota and adult hippocampal neurogenesis. Cell Reports 15: 1945-1956.

Nimgampalle, M. and Kuna, Y., 2017. Anti-Alzheimer properties of probiotic, Lactobacillus plantarum MTCC 1325 in Alzheimer's disease induced albino rats. Journal of Clinical and Diagnostic Research 11: KC01-KC05.

O’Hagan, C., Li, J.V., Marchesi, J.R., Plummer, S., Garaiova, I. and Good, M.A., 2017. Long-term multi-species Lactobacillus and Bifidobacterium dietary supplement enhances memory and changes regional brain metabolites in middle-aged rats. Neurobiology of Learning and Memory 144: 36-47.

Park, J., Lee, J., Yeom, Z., Heo, D. and Lim, Y.H., 2017. Neuroprotective effect of Ruminococcus albus on oxidatively stressed SH-SY5Y cells and animals. Scientific Reports 7: 14520.

Parsons, R.B., Aravindan, S., Kadampeswaran, A., Evans, E.A., Sandhu, K.K., Levy, E.R., Thomas, M.G., Austen, B.M. and Ramsden, D.B., 2011. The expression of nicotinamide $\mathrm{N}$-methyltransferase increases ATP synthesis and protects SH-SY5Y neuroblastoma cells against the toxicity of Complex I inhibitors. Biochemical Journal 436: 145-155.

Pervin, M., Unno, K., Nakagawa, A., Takahashi, Y., Iguchi, K., Yamamoto, H., Hoshino, M., Hara, A., Takagaki, A., Nanjo, F., Minami, A., Imai, S. and Nakamura, Y., 2017. Blood brain barrier permeability of (-)-epigallocatechin gallate, its proliferationenhancing activity of human neuroblastoma SH-SY5Y cells, and its preventive effect on age-related cognitive dysfunction in mice. Biochemistry and Biophysics Reports 9: 180-186.

Proia, P., Di Liegro, C.M., Schiera, G., Fricano, A. and Di Liegro, I., 2016. Lactate as a metabolite and a regulator in the central nervous system. International Journal of Molecular Sciences 17: E1450. 
Sarkar, A., Lehto, S.M., Harty, S., Dinan, T.G., Cryan, J.F. and Burnet, P.W., 2016. Psychobiotics and the manipulation of bacteria-gut-brain signals. Trends on Neuroscience 39: 763-781.

Umbrello, G. and Esposito, S., 2016. Microbiota and neurologic diseases: potential effects of probiotics. Journal of Translational Medicine 14: 298.

Wahl, D., Coogan, S.C., Solon-Biet, S.M., De Cabo, R., Haran, J.B., Raubenheimer, D., Cogger, V.C., Mattson, M.P., Simpson, S.J. and Le Couteur, D.G., 2017. Cognitive and behavioral evaluation of nutritional interventions in rodent models of brain aging and dementia. Clinical Interventions in Aging 12: 1419-1428.

Wang, Y., Wu, Y., Xu, H., Mei, X., Yu, D. and Li, W., 2017. Antioxidant properties of probiotic bacteria. Nutrients 9: 521.
Westfall, S., Lomis, N., Kahouli, I., Dia, S.Y., Singh, S.P. and Prakash, S., 2017. Microbiome, probiotics and neurodegenerative diseases: deciphering the gut brain axis. Cellular and Molecular Life Sciences 74: 3769-3787.

Winner, B. and Winkler, J., 2015. Adult neurogenesis in neurodegenerative diseases. Cold Spring Harbor Perspectives in Biology 7: a021287.

Wyss-Coray, T., 2016. Ageing, neurodegeneration and brain rejuvenation. Nature 539: 180-186.

Xicoy, H., Wieringa, B. and Martens, G.J., 2017. The SH-SY5Y cell line in Parkinson's disease research: a systematic review. Molecular Neurodegeneration 12: 10. 
